\title{
Le futur du transport est-il technologique ? Quelles innovations pour quelles évolutions du système de transport?
}

\author{
Is technology the only future for transportation? Which innovations for \\ which changes in the transportation system?
}

\author{
Corinne Blanquart ${ }^{1}$ \\ ${ }^{1}$ Université Paris-Est, IFSTTAR, SPLOTT, France, corinne.blanquart@ifsttar.fr
}

\begin{abstract}
RÉSUMÉ. Le présent dossier illustre la nécessité d'éclairer l'ensemble des innovations possibles dans le champ du transport et de la logistique, innovations de services, mais aussi innovations organisationnelles, au-delà des seules innovations technologiques. En considérant le système de transport comme un système socio-technique, il montre l'intérêt d'analyser les conditions de production des services, dans le cadre de relations entre les acteurs de ce système, et ce faisant les conditions de production des innovations de services. Ces dernières apparaissent dès lors comme des éléments essentiels des caractéristiques mais aussi de l'influence des innovations en question.

ABSTRACT. This special issue illustrates the need to highlight all the possible innovations in the field of transport and logistics: service innovations, and also organizational innovations, beyond technological innovations alone. By considering the transport system as a socio-technical system, it shows the interest of considering the conditions of services production, based on relationships between the actors of the system, as well as the conditions of production of services innovations. The latter appear therefore as essential elements of the characteristics but also of the influence of the innovations in question.

MOTS-CLÉS. Innovations, transport, logistique, services, organisations.

KEYWORDS. Innovations, transport, logistics, services, organizations.
\end{abstract}

\section{Introduction}

L'innovation a de longue date été un levier majeur de politiques publiques dans le champ du transport et de la logistique. Le rapport de la commission nationale d'évaluation des politiques d'innovation atteste que « le soutien public à l'innovation (Etat, régions, Europe...) a doublé, pour atteindre les 10 milliards d'euros, soit un demi-point de PIB» (2016). 6 milliards d'euros sont consacrés au seul programme européen de recherche Horizon 2020, destiné à promouvoir des projets de transport durable.

Si l'ensemble des programmes d'innovation se déploient sous la bannière du transport durable, les externalités environnementales associées au transport demeurent, en lien avec la domination du mode routier, et ce malgré l'importance des budgets consacrés. Les effets attendus des innovations soutenues et déployées sont donc à nuancer.

Les effets discutables des innovations mises en œuvre amènent à questionner la pertinence de ces innovations, majoritairement technologiques. A l'heure du véhicule autonome, des systèmes de transport intelligents reposant sur la diffusion des TIC au transport ou du déploiement du numérique, l'effort est en effet porté sur l'innovation technologique. Les pôles de compétitivité dédiés au transport ou les appels à propositions de recherche font ainsi la part belle aux technologies innovantes. Or, un système de transport articule certes des systèmes techniques (véhicules, infrastructures, TIC), mais aussi des systèmes d'acteurs (opérateurs, usagers, constructeurs, collectivités). En portant l'accent sur les systèmes techniques, on néglige ce faisant la dimension de service du transport, soutenant la mise en œuvre d'un service de mobilité à destination des usagers, à la demande d'autorités organisatrices, et mis en œuvre par des opérateurs de transport. L'organisation de ce système d'acteurs, et ses évolutions, est également éludée. Par ailleurs, et y compris sur le versant des innovations technologiques, la 
permanence des nuisances environnementales du transport peut plaider en faveur d'innovations plus radicales.

Il apparaît donc nécessaire d'éclairer l'ensemble des innovations possibles dans le champ du transport et de la logistique, innovations de services, mais aussi innovations organisationnelles, au-delà des seules innovations technologiques. Il semble également nécessaire de considérer les innovations radicales à mêmes d'inverser la domination du mode routier source d'externalités environnementales.

Considérer le transport comme un système socio-technique permet par ailleurs de s'intéresser aux conditions de production des services, dans le cadre de relations entre les acteurs de ce système, et ce faisant aux conditions de production des innovations de services. Ces dernières apparaissent dès lors comme des éléments essentiels des caractéristiques mais aussi de l'influence des innovations en question.

La démarche amène alors à délaisser une approche déterministe en termes d'effets des technologies pour s'orienter vers une analyse des interactions entre des formes d'organisation du transport et des systèmes productifs et territoriaux. L'impact potentiel des systèmes de transport intelligent a été apprécié tant au niveau de la recherche que des premières phases de déploiement. De nombreux travaux s'attachent à l'analyse de l'évolution des caractéristiques du déplacement (coût, temps, répartition spatiale des trafics). Toutefois, ces résultats sont conditionnés par la mobilisation des services, que soutiennent les systèmes de transport intelligent. La réponse en termes d'offre technologique, de la même façon que celle en termes d'infrastructures, doit être pensée en fonction des besoins, de la demande des usagers. Or, ces besoins apparaissent très divers.

Car ce sont bien les effets de ces innovations qui sont discutés, voire mis en cause par la permanence des externalités environnementales, ou par la difficulté de faire émerger une influence homogène de ces innovations.

Or, les choix d'innovation sont souvent dictés par la comparaison de bonnes pratiques issues d'autres pays ou d'autres territoires. L'exemple de la logistique urbaine est à cet égard illustratif, objet de nombreux benchmarks des initiatives mises en place en Europe, en vue de leur réplication dans d'autres pays. Le projet Interreg SUGAR (Sustainable Urban Goods logistics Achieved by Regional and local policies) était ainsi centré sur la transmission des savoirs et les bonnes pratiques. Des innovations de logistique urbaine étaient ainsi valorisées, dont le centre de distribution urbaine de Motomachi au Japon. Les centres de distribution urbaine qui se sont développés en France à partir des années 2000 n'ont pour autant pas su faire la preuve de leur rentabilité, et corrélativement pas pu être pérennisés pour la plupart.

Il est donc là encore important d'éclairer les conditions de production différenciées dans le temps et l'espace des innovations logistiques et de transport, au regard du système d'acteurs et d'institutions. La prestation se présente en effet comme un construit social entraînant une diversité des situations de transport dans le temps et l'espace, en fonction des modalités de la coordination située entre les acteurs. L'analyse de la coordination située permet de revenir plus largement sur la place de l'espace dans l'analyse, souvent réduit à une contrainte, une friction, caractérisable en termes de temps, et de coûts. Dans cette représentation, le transport permet de desserrer la contrainte. Mais les interactions entre acteurs localisés dans l'espace articulent des registres variés, qui offrent ainsi un cadre plus riche de lecture de l'espace. La proximité géographique des acteurs ne détermine pas à elle seule les interactions, ni leur qualité. Ainsi, si le transport influe sur les proximités géographiques, elles ne suffisent pas à expliquer la dynamique de fonctionnement des territoires.

Le présent numéro spécial aborde l'ensemble des types d'innovation dans le champ de la logistique et du transport, incluant les innovations technologiques, mais aussi de services (les services de transport à grande vitesse), ou organisationnelles (les innovations dans les organisations logistiques associées au développement du e-commerce). Il traite d'innovations incrémentales mais pose également la question d'innovations radicales, pour endiguer notamment le déclin du fret ferroviaire. 
Les innovations décrites concernent à la fois le transport de marchandises (l'überisation de la livraison du dernier kilomètre) ou la mobilité des personnes (les services ferroviaires à grande vitesse). Enfin, elles portent sur les deux principaux modes de transport terrestres, ferroviaire et routier.

L'enjeu de ce numéro spécial est de montrer que les conditions de production des innovations conditionnent leur émergence, leurs caractéristiques et leur influence. Plusieurs conditions sont ainsi mises en avant:

- les différences de business models ; l'article de Christine Belin-Munier montre que les composants des business models permettent de différencier les choix stratégiques des entreprises, notamment concernant les services logistiques associés au e-commerce; ces business models sont alors décrits dans leur variété comme des sources d'innovations ;

- les caractéristiques du secteur et notamment les modalités de coordination entre les acteurs; l'article de Raimbault et alii propose ainsi une analyse du système d'innovation dans le ferroviaire en termes de système sectoriel d'innovation et de production ;

- les ressources et les institutions entendues en tant que systèmes de valeurs et de règles; l'article de Marie Delaplace illustre qu'elles influencent les caractéristiques techniques du service ferroviaire à grande vitesse, mais aussi l'appropriation par les usagers de ce service.

Ces conditions renvoient à plusieurs échelles pour l'analyse des déterminants de l'innovation : celle micro-économique du projet d'innovation, celle méso-économique du système d'acteurs et de sa structure, celle enfin macro-économique des institutions et du contexte économique général. L'article de Müller et Blanquart montre l'articulation de ces trois niveaux de déterminants pour expliquer les différences entre la France et l'Allemagne dans l'émergence d'innovations radicales pour le transport de marchandises.

En effet, l'entrée par les conditions de production de l'innovation permet d'expliquer les différences observées entre les pays ou entre les territoires dans les caractéristiques des innovations rencontrées, comme dans leurs effets.

Les effets de l'überisation de la logistique en termes de fragmentation spatiale à l'échelle de l'Ile de France sont ainsi décrits par Vétois et Raimbault, avec une grande logistique périurbaine non überisée, et une logistique du dernier kilomètre überisée. Cette fragmentation spatiale aboutit à une fragmentation sociale, illustrant ce faisant les effets parfois négatifs des innovations.

L'article de Marie Delaplace montre aussi l'hétérogénéité entre les territoires des supposés « effets $\mathrm{TGV} »$ au regard des contextes d'institutions et de ressources dans lesquels sont produits les services ferroviaires à grande vitesse.

Il montre également l'influence de ces contextes sur les caractéristiques du service ferroviaire mis en place.

Cette analyse des conditions de production des innovations explique enfin les difficultés d'émergence même des innovations ferroviaires, en éclairant comme dans l'article de Raimbault et alii des modalités de coordination des acteurs du système ferroviaire qui n'ont pas encore tout à fait suivi l'évolution des règles de concurrence apportées par les directives de libéralisation. A l'échelle européenne, des divergences dans l'émergence et la diffusion ou non d'innovations radicales entre la France et l'Allemagne sont également éclairées dans l'article de Müller et Blanquart au regard des contextes micro, méso et macro dans lesquels émergent les projets.

Le numéro spécial est articulé comme suit, en montrant successivement les déterminants:

- de l'émergence même des innovations, au travers de l'article de Raimbault et alii sur les difficultés d'innovation en matière ferroviaire ; 
- des caractéristiques des innovations observées : radicales pour l'article de Müller et Blanquart, de services ferroviaires à grande vitesse pour l'article de Marie Delaplace, d'organisations logistiques du e-commerce pour l'article de Christine Belin-Munier ;

- des effets de ces innovations, au travers de l'exemple décrit par Vétois et Raimbault autour de l'überisation de la logistique urbaine.

Ce faisant, ce numéro spécial offre un cadre d'analyse des innovations en matière de transport qui dépasse les grilles de lecture en termes d'effets. Il prône une analyse systémique des innovations logistiques et de transport, considérant conjointement le système technique et le système d'acteurs, tant pour renouveler les modalités de production et de diffusion des innovations que pour analyser leur influence. Envisager enfin le système de transport comme un système socio-technique amène à rééquilibrer la place des innovations non technologiques en matière de transport, qu'elles soient servicielles ou organisationnelles. Le Programme de REcherche et D'Innovation dans les Transports terrestres (PREDIT) œuvrait en ce sens, en accordant au sein de ses groupes opérationnels une place à la recherche et à l'innovation non technologiques. Gageons qu'un programme similaire prendra sa suite. 